\title{
The Role of Adrenal Cortex-Sparing Surgery for Bilateral Masses in Three Cases
}

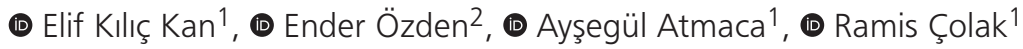 \\ 1 Ondokuz Mayıs University Faculty of Medicine, Department of Endocrinology and Metabolism, Samsun, Turkey \\ 2 Ondokuz Mayıs University Faculty of Medicine, Department of Urology, Samsun, Turkey
}

\begin{abstract}
Pheochromocytoma are the functional adrenal lesions originating from the chromaffin cells. For the cases of pheochromocytoma observed in multiple endocrine neoplasia Type 2 and Von Hippel syndrome, the bilateral adrenal glands are involved. In classical approach, total adrenalectomy is applicable on such masses while adrenal failure is almost inevitable. Lifelong cortisol and fludrocortisones replacement are necessary for the patients with adrenal failure while the rate of morbidity and mortality has significantly increased. With the introduction of the minimal invasive surgical approach, cortex sparing adrenalectomy has been brought forward for the adrenal tumors. The primary objective of the cortex sparing surgery is to prevent the lifelong replacement and the permanent adrenal failure after adrenalectomy. Therefore, it is particularly preferred in the case of genetic pheochromocytoma with bilateral adrenal involvement. However, in the case of the selected cases, it can also be applicable for adenoma producing aldostrerone and Cushing syndrome. The adrenal tumor will be completely removed and if sufficient tissue is reserved in the manner to preserve the cortex function, no long-term recurrence and adrenal failure is to be developed. Therefore, cortex-sparing surgery may be a good alternative to total adrenalectomy for the patients with small benign functional adrenal tumors or bilateral genetic pheochromocytoma.
\end{abstract}

Keywords: Pheochromocytoma, MEN 2A, VHL syndrome, partial adrenalectomy

\section{Introduction}

Pheochromocytoma are the neuroendocrine tumors originated from chromaffin cells of the adrenal gland. Multiple endocrine neoplasm type 2 (MEN 2), Von Hippel Lindau (VHL) and Von Recklinghausen (neurofibromatosis type 1) syndromes are the genetic diseases associated with the pheochromocytoma. Pheochromocytoma observed with such syndromes frequently retain the bilateral adrenal glands. The classical approach to adrenal masses is total adrenalectomy. However, in the case of bilateral adrenal involvement, the occurrence of adrenal failure is inevitable subsequent to adrenalectomy and lifelong cortisol replacement is a must. Despite the appropriate replacement of the hormones, mortality risks increase due to Addison crisis. Partial adrenalectomy option has been brought forward to preserve the functions of the adrenal cortex as a consequence in the development of the minimal invasive surgical interventions. Different terminology is applied for non-total adrenalectomy in the manner to include "sub-total adrenalectomy", "cortex sparing adrenalectomy", "partial adrenalectomy" or "adrenal sparing adrenalectomy" in the literature (2). Laparoscopic cortex sparing surgery for bilateral adrenal tumors is a recently introduced technique and it provides advantages to the patients that no adrenal failure is developed and no hormone replacement is deemed to be necessary. The widely used indications for this particular procedure are the syndromic diseases where the bilateral adrenal glands are impacted such as MEN 2A and 2B, VHL, Neurofibromatosis (3).

In this study, we aimed to summarize the role of bilateral adrenal cortex sparing surgery which is a recently introduced technique in the light of 3 cases.

\section{Case 1}

Male patient aged 39 years who was examined for hypertension attack was diagnosed with pheochromocytoma in 2012. The dynamic magnetic resonance (MR) imaging through adrenal protocol revealed bilateral non-adenoma lesions with limited diffusion. These lesions were described as $11 \mathrm{~mm}$ on the right adrenal body, $15 \mathrm{~mm}$ on the lateral adrenal crus, and $14 \mathrm{~mm}$ on the left adrenal. On the other hand, thyroid ultrasonography showed nodules with a size of $10 \times 6 \mathrm{~mm}$ in the right lobe and a size $9 \times 8 \mathrm{~mm}$ in the left lobe. The fine needle aspiration biopsy of these thyroid nodules revealed medullar carcinoma. The patient was diagnosed as MEN2A based on these findings. No parathyroid adenoma was found. In the genetic examination

Cite this article as: Kılıç Kan E, Özden E, Atmaca A, Çolak R. The Role of Adrenal Cortex-Sparing Surgery for Bilateral Masses in Three Cases. Bull Urooncol 2019;18(4):42-46 
Cys634Arg heterozygote mutation was determined and genetic consultation was suggested for the first degree relatives. Left partial adrenalectomy and right total adrenalectomy were performed and the bilateral adrenal veins were resected. The postoperative urinary catecholamines were found normal. The postoperative urinary catecholamine was found to be normal. Also, the patient underwent total thyroidectomy and bilateral neck dissection by general surgeons. The pathology reported bilateral thyroid medullar carcinoma. The patient has been followed up for 72 months without the need for cortisol and fludrocortisone replacement. Patient was normotensive while the annual catecholamine levels were found to be normal. Levothyroxine replacement was given and the follow up continues for thyroid medullar carcinoma.

\section{Case 2}

A female patient, aged 43, was diagnosed with hypertension three years ago. She was under follow up at the cardiology clinic for persistent hypertension where carvedilol benidipine, spirinolactone + hydrochlorothiazide treatments were given. Upon being understood that her brother had MEN2A diagnosis, the upper abdomen computerized tomography (CT) was performed. CT revealed that bilateral adrenal lesions on the left and right adrenal glands, dimensioned as $57 \times 53 \mathrm{~mm}$ and $16 \times 10 \mathrm{~mm}$, respectively. In her medical history, it was found out that she underwent parathyroid adenoma operation with total thyroidectomy in 2006. The pathologies were inaccessible. The calcitonin levels was found to be $80.6 \mathrm{pg} / \mathrm{mL}$ which was asked for the potentiality of the medullar thyroid carcinoma. No pathologic finding was determined in cervix ultrasonography. Cys634Arg heterozygote mutation was detected in the genetic examination asked for the RET proto-oncogene. 24-hour urinary catecholamines were found high. I-123 MIBG and SPECT-CT were performed with the diagnosis of bilateral pheochromocytomas. MIBG positive lesions were detected in $60 \mathrm{~mm}$ diameter lesion located on the left adrenal gland and 30 $\mathrm{mm}$ lesion on the right adrenal gland. Upon completion of the anesthesia preparations in an appropriate manner, laparoscopic left adrenalectomy was performed in the first operation and partial adrenalectomy was carried out in the second operation. The patient who has been under control postoperatively for 4 months, has been followed up without the use of the antihypertensive and with no need for cortisol.

\section{Case 3}

Forty-two year old man admitted to hospital with epigastric pain. Lower abdominal MR revealed $19 \times 17 \mathrm{~mm}$, oval, hyperintense lesion at segment- 2 of the liver, non-adenoma lesions dimensioned to be $97 \times 73 \times 62 \mathrm{~mm}$ on the right adrenal, $21 \times 19 \mathrm{~mm}$ on the left adrenal crus, $24 \times 23 \mathrm{~mm}$ cortical cysts on the right kidney (Figure 1A). As a consequence of the endocrine assessments, the patient whose 24h urinary catecholamine was responsive for pheochromocytoma, underwent partial nephrectomy and total adrenalectomy for the right side subsequent to anesthesia preparation conducted in an appropriate manner. The pathology reported clear renal cell carcinoma, while the adrenal pathology revealed hyalinization and myxoid changes in stroma and the findings not encountered in sporadic pheochromocytoma such as the capsule with vascularized thick wall and local oval shaped nucleus cell. After the first operation, the patient with high catecholamine values underwent partial adrenalectomy with preserving the left adrenal vein within 6 months subsequent to the first operation. The limits of the mass was defined through ultrasonography in laparoscopic transperitoneal approach (Figure 2). After applying indo cyanine green injection, the mass and the adrenal tissues were disentangled. The mass was excised leaving aside some sound adrenal tissue (Figure 3). The postoperative MR showed no adrenal gland on the right side but excised mass on the left adrenal and the presence of adrenal tissues (Figure 1B). The urinary catecholamine of the patient after eleven months was normal and the patient has been followed up with no need for cortisol.

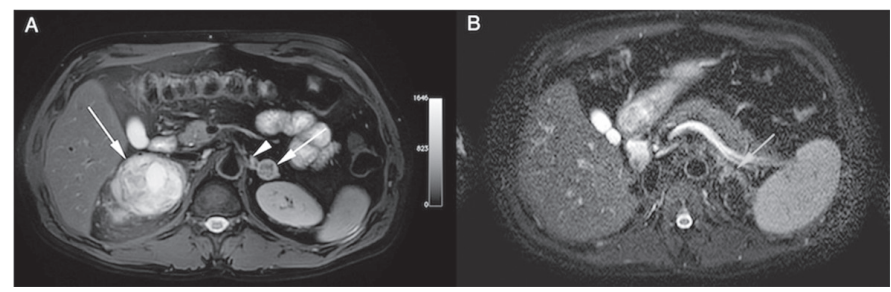

Figure 1A. Magnetic resonance image of the postoperative bilateral adrenal mass. B. Postoperative magnetic resonance image

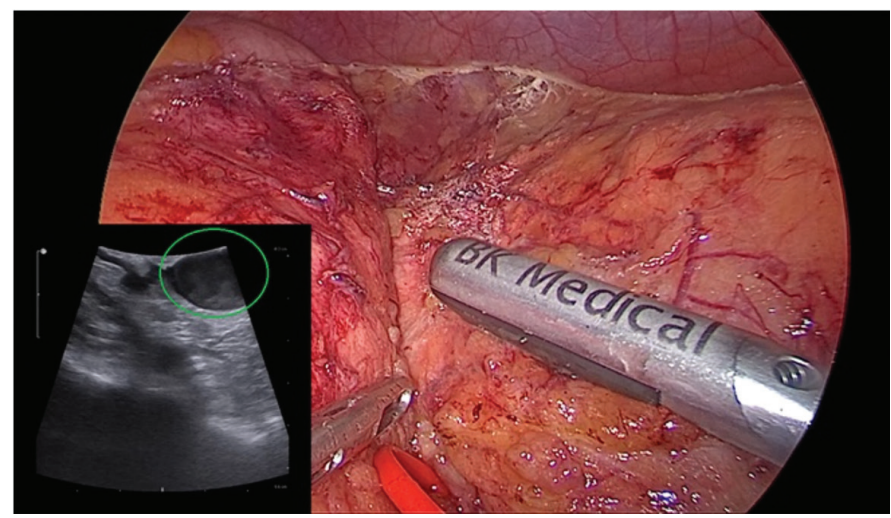

Figure 2. Determination of the borders of the mass through intraoperative ultrasonography during partial adrenalectomy for the left adrenal

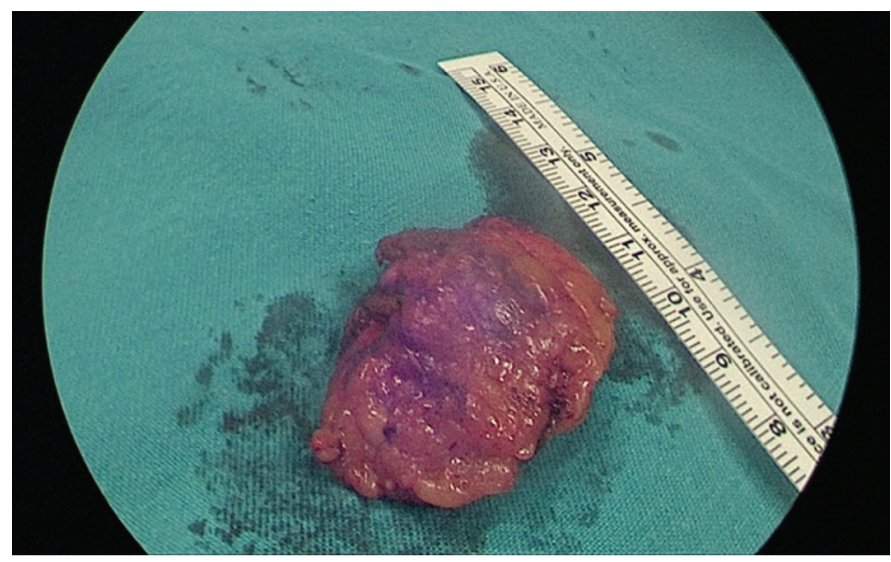

Figure 3. Specimen taken subsequent to partial adrenalectomy for the left adrenal 


\section{Discussion}

Laparoscopic transperitoneal adrenalectomy was defined by Gagner et al. in (4). It is the golden standard procedure for adrenalectomy due to its high rate of achievement and low morbidity advantages. Optically increased tumor imaging, decreased pain and recovery time, short hospitalization time and improved cosmetic results are the advantages of laparoscopic adrenalectomy. On the contrary, laparoscopic technique disposes the tactile sensing used traditionally by the surgeon for the purpose of distinguishing the boundaries of the mass and defining the vascular structures. For the syndromic diseases with bilateral adrenal gland involvement, in case bilateral total adrenalectomy is applicable through classical approach, development of adrenal failure is inevitable. The probability of the additional surgeries is higher for such syndromes due to the components other than the pheochromocytoma and the protection of the endogenous corticosteroid sources is of particular importance. Therefore, partial adrenalectomy is the appropriate option for these patients. Although the indications of the cortex sparing surgery have not yet been clearly defined, the widely accepted indication is the bilateral pheochromocytoma due to the hereditary diseases. Cortex sparing or partial adrenalectomy may be applicable through open surgery, transperitoneal laparoscopic or retroperitoneal methods (5). The points which should must be taken into consideration in cortex sparing adrenalectomy are full excision of the adrenal mass and leaving off the tissue sufficient to preserve the cortex functionality. It has been reported in the contents of the study by Casinetti et al. (6) that although almost all of the patients who underwent bilateral adrenalectomy were in need of cortisol replacement, $57 \%$ of the patients who underwent cortex sparing surgery did not require any cortisol. The primary question about the cortex sparing surgery for bilateral pheochromocytoma is the risk of recurrence due to the fact that the resection of the medullar tissues cannot always be verified in a definite manner. The risk of recurrence for hereditary bilateral pheochromocytoma is higher than sporadic pheochromocytoma. One of the studies evidenced that the rate of recurrence for the patients with hereditary pheochromocytoma is $20 \%$ and the rate of patients who require steroid replacement is $8 \%$ (7). A study by Kaye et al. (8) revealed that when 22 series which include 417 patients are examined, $76 \%$ of the applicable procedure is laparoscopic, only $3 \%$ of the patients showed recurrent lesions by the end of the 56 months follow up period and $5.3 \%$ of the patients required steroid in the long term. When the study related to the data concerning 563 patients who underwent partial adrenalectomy upon being diagnosed with pheochromocytoma based on MEN 2 syndrome, $3 \%$ of the adrenal glands on which cortex sparing adrenalectomy was applied showed recurrence, while $2 \%$ of the glands with total adrenalectomy showed recurrence. The difference was not significant. It has been found out that the average recurrence period subsequent to cortex sparing surgery is 9.5 years. In the previous studies, the rate of recurrence subsequent to cortex sparing surgery was $21 \%$, while the rates of recurrence after laparoscopic adrenalectomy are less than 5\% (6). In a prospective study concerning 6 patients with bilateral adrenal involvement, retroperitoneal cortex sparing surgery was applied. After a follow up period of $26 \pm 6$ months, none of the patients have developed any recurrence and 5 of the patients (83\%) required any cortisol (9). Laparoscopic cortex sparing adrenalectomy was applicable in 3 of our cases and none of the cases developed recurrent pheochromocytoma.

One of the syndromes for which cortex sparing surgery may be applicable is the VHL syndrome. VHL syndrome is autosomal dominant hereditary disease and encountered at a rate of $1: 36.000$. Out of the widely encountered tumors accompanying to the $\mathrm{VHL}$ disease are the central nervous system hemangioblastoma, clear cell renal carcinoma, pancreas neuroendocrine tumor, tympanum endolymphatic sac tumors and pancreas cysts (10). The rate of bilateral adrenal involvement for the VHL patients varies between $40 \%$ and $80 \%$. Even though there may be clinically unilateral involvement by the time of diagnosis in MEN 2 or VHL syndromes, unilateral cortexsparing surgery is recommended since the risk of involvement of the other adrenal gland is too high (11).

The other group of disease for which cortex sparing surgery is recommended is the Conn syndrome. The study examining the prospective results of the total and partial adrenalectomy on 212 patients suffering Conn syndrome with unilateral adenoma evidence that the serum aldosterone results of all of the patients are normalized subsequent to unilateral partial or total adrenalectomy and no substantial difference has been observed between the two approaches in view of the number of patients which should have antihypertensive treatment (12). Unilateral cortex sparing surgery is the preferred method for Conn syndrome based on the fact that no adrenal failure develops in the case of occurrence of any subsequent influential disease. It is also due to the benignity of the aldosterone secreting adenoma, anatomical responsiveness for cortex sparing surgery and similar rate of treatment wise achievement of both cortex sparing surgery and total adrenalectomy.

Partial adrenalectomy may also be applicable for Cushing syndrome. The study conducted by He et al. (13), 87 patients who underwent partial adrenalectomy showed a treatment success at a rate of $97.8 \%$. During partial adrenalectomy for the patients with Cushing syndrome, the surrounding adipose tissue should be removed for the clear detection of the adenoma limits, distinction of the normal tissues in an absolute manner and resection of adenoma to give way to a minimum $5 \mathrm{~mm}$ normal tissue particles (14). However, partial adrenalectomy may technically be difficult for the patients with Cushing syndrome due to the excessive retroperitoneal and the adipose tissues in the vicinity of the adrenal gland. Intraoperative ultrasonography may apply in cases where it is difficult to distinguish the boundaries of the normal tissue with the adrenal mass.

It is considered that adrenal vein should be preserved in the course of the adrenal cortex sparing surgery theoretically, while it is not a requisite to preserve the adrenal vein for the purpose of the sufficient postoperative cortical functioning. The other problem is that the minimum amount of adrenal tissue necessary for normal physiology is still not known. Brauckhoff et al. (15) have reviewed 10 patients who underwent subtotal adrenalectomy where less than one third of the adrenal gland was preserved with the transection of the main adrenal vein. 
To verify the adrenal functioning during postoperative period, they have made adrenocorticotropic hormone test and they have concluded that venous drainage through the main vein is not critical for adrenocortical functions and that an estimated quantity of one third of the adrenal tissue is sufficient for adrenal functioning. The anatomic study to document the adrenal blood feeding regarding the laparoscopic partial adrenalectomy showed the numerous small veins around the adrenal gland and it is suggested that no aggressive adrenal gland mobilization should be made in order not to destruct the venous small veins (16). Meanwhile, if not adjacent to the tumor, it is recommended to keep the main venous drainage intact. In addition, the collagen tissues remaining around the adrenal tissue should be kept safe to prevent likely torsion and preserve the arterial feeding. After the cortex sparing surgery, minimum $1 / 3$ of the unimpaired cortex tissue should be preserved for the continuance of the usual cortical functions. We have also executed partial adrenalectomy in 2 of our cases including a patient who is under follow-up for 72 months, with no preservation of the adrenal vein. and no pathology has been observed in the functions of the adrenal gland.

For the purpose of successful laparoscopic adrenalectomy, the proper definition of the anatomy is an important step. Right adrenal vein is short and connected to the inferior vena cava vein directly, while left adrenal vein is longer and discharges to the renal vein. The detection of the adrenal vein in a swift and careful manner is particularly important for the resection of the pheochromocytoma due to the hemodynamic stress observed in tumor manipulation. The surgeries carried out under the guidance of the fluorescence may provide precious data in view of the dissection and progresses of the critical vascular structures. The intensity of the fluorescent signals make the lesions and vein more often within the robusting organs in the adjacent tissues such as adrenal gland, visible. Indio cyanine green (ICG) is a non-toxic paint approved by FDA which spread away the close infrared $(600-900 \mathrm{~mm})$ fluorescent signals in imaging. The optimal dose which should be used has been defined while the maximum recommended dose is $2 \mathrm{mg} / \mathrm{kg}$ for the adults with normal liver function tests (17). Even if the diluted ICG used during the laparoscopic process supported by fluorescent is applied repeatedly, it is far below the recommended dosage. Therefore, whenever ICG dose is required, it may be repeated safely during the process. Pheochromocytoma, malign adrenal tumors and adrenal cortical neoplasia are hypo fluorescent. Although imaging through ICG is not superior to the conventional robotic imaging for such types of adrenal masses in view of the boundaries of the masses, it is much more superior for the hyperfluorescent masses (18). The fact that the failure to image some of the tumors such as pheochromocytoma, adrenal cortical neoplasia/carcinoma through fluorescent is deemed to be the a throwback. In addition, it may provide additional efficiency for adrenal sparing surgeries in view of imaging the usual borders of the tissues and the hypo fluorescent mass.

As a consequence, the most important points which should be taken into consideration in partial adrenalectomy are the full resection of the adrenal masses and additionally, leaving aside tissues sufficient to preserve the functioning of the cortex.
In particular it should be considered as the method which should be preferred in the first priority due to less morbidity in the syndromic cases with adrenal gland involvement and no necessity for long term replacement.

\section{Acknowledgements}

Publication: The results of the study were not published in full or in part in form of abstracts.

Contribution: There is not any other contributors who may not be listed as authors.

Conflict of Interest: No conflict of interest was declared by the authors.

Financial Disclosure: The authors declared that this study received no financial support.

\section{Ethics}

Informed Consent: All patients read the patient information form and written informed consents were obtained.

Peer-review: Externally peer-reviewed.

\section{Authorship Contributions}

Design: E.K.K., Data Collection or Processing: E.K.K., E.Ö., Analysis or Interpretation: E.Ö., Literature Search: A.A., R.Ç., Writing: E.K.K.

\section{References}

1. Husebye ES, Allolio B, Arlt W, et al. Consensus statement on the diagnosis, treatment and follow-up of patients with primary adrenal insufficiency. J Intern Med 2014;275:104-115.

2. Madala A, Daugherty M, Bratslavsky G. Partial Adrenalectomy. Why Should it be Considered? Urology Practice 2015;2:359-366.

3. Walz MK. Extent of adrenalectomy for adrenal neoplasm: cortical sparing (subtotal) versus total adrenalectomy. Surg Clin N Am 2004;84:743-753.

4. Gagner M, Lacroix A, Bolte E. Laparoscopic adrenalectomy in Cushing's syndrome and pheochromocytoma. N Engl J Med 1992;327:1033.

5. Conzo G, Tartaglia E, Gambardella C, et al. Minimally invasive approach for adrenal lesions: Systematic review of laparoscopic versus retroperitoneoscopic adrenalectomy and assessment of risk factors for complications. Int J Surg 2016;28:118-123.

6. Castinetti F, Qi XP, Walz MK, et al. Outcomes of adrenal-sparing surgery or total adrenalectomy in phaeochromocytoma associated with multiple endocrine neoplasia type 2: an international retrospective population-based study. Lancet Oncol 2014;15:648-655.

7. Lee JE, Curley SA, Gagel RF, et al. Cortical sparing adrenalectomy for patients with bilateral pheochromocytoma. Surgery 1996;120:1064-1071.

8. Kaye DR, Storey BB, Pacak K, et al. Partial adrenalectomy underused first line therapy for small adrenal tumors. J Urol 2010;184:18-25.

9. Vidal O, Delgado-Oliver E, Diaz del Gabbo R, et al. Functional Adrenal Cortex Preservation: A Good Reason for Posterior Retroperitoneal Endoscopic Approach. Cır Esp 2018;96:488-493.

10. Lonser RR, Glenn GM, Walther M, et al. von Hippel-Lindau disease. Lancet 2003;361:2059-2067.

11. Inabnet WB, Caragliano P, Pertsemlidis D. Pheochromocytoma: inherited associations, bilaterality, and cortex preservation. Surgery 2000;128:1007-1011. 
12. Fu B, Zhang X, Wang G, et al. Long-Term Results of a Prospective, Randomized Trial Comparing Retroperitoneoscopic Partial Versus Total Adrenalectomy for Aldosterone Producing Adenoma. The J Urol 2011;185:1578-1582.

13. He HC, Dai J, Shen Z, et al. Retroperitoneal Adrenal-Sparing Surgery for the Treatment of Cushing's Syndrome Caused by Adrenocortical Adenoma:8-Year Experience With 87 Patients. World J Surg 2012;36:1182-1188.

14. Walz MK, Peitgen K, Diesing D, et al. Partial versus Total Adrenalectomy by the Posterior Retroperitoneoscopic Approach: Early and Longterm Results of 325 Consecutive Procedures in Primary Adrenal Neoplasia. World J Surg 2004;28:1323-1329.

15. Brauckhoff M, Gimm O, Thanh PN, et al. Critical size of residual adrenal tissue and recovery from impaired early postoperative adrenocortical function after subtotal bilateral adrenalectomy. Surgery 2003;134:1020-1027.

16. Parnaby CN, Galbraith N, O'Dwyer PJ. Importance of the adrenal gland blood supply during laparoscopic subtotal adrenalectomy. J Laparoendosc Adv Surg Tech A 2010;20:311-315.

17. Marshall MV, Rasmussen JC, Tan IC, et al. Near-infrared fluorescence imaging in humans with indocyanine green: A review and update. Open Surg Oncol J 2010;2:12-25.

18. Colvin J, Zaidi N, Berber E. The utility of indocyanine green fluorescence imaging during robotic adrenalectomy J Surg Oncol 2016;114:153-156. 